\title{
Review of Distribution Network Phase Unbalance: Scale, Causes, Consequences, Solutions, and Future Research Direction
}

\author{
Kang Ma, Lurui Fang, Wangwei Kong
}

\begin{abstract}
Phase unbalance is widespread in the distribution networks in the UK, continental Europe, US, China, and other countries. First, this paper reviews the mass scale of phase unbalance and its causes and consequences. Three challenges arise from phase rebalancing: the scalability, data scarcity, and adaptability (towards changing unbalance over time) challenges. Solutions to address the challenges are: 1) using retrofit-able, maintenance-free, automatic solutions to overcome the scalability challenge; 2) using data analytics to overcome the data-scarcity challenge; and 3) using phase balancers or other online phase rebalancing solutions to overcome the adaptability challenge. This paper categorizes existing phase rebalancing solutions into three classes: 1) load/lateral re-phasing; 2) using phase balancers; 3) controlling energy storage, electric vehicles, distributed generation, and micro-grids for phase rebalancing. Their advantages and limitations are analyzed and ways to overcome the limitations are recommended. Finally, this paper suggests future research topics: 1) long-term forecast of phase unbalance; 2) whole-system analysis of the unbalance-induced costs; 3 ) phase unbalance diagnosis for data-scarce LV networks; 4) technocommercial solutions to exploit the flexibility from large threephase customers for phase balancing; 5) the optimal placement of phase balancers; 6) the transition from single-phase customers to three-phase customers.
\end{abstract}

Index Terms-low voltage, power distribution, three-phase system, phase unbalance, phase balancing

\section{INTRODUCTION}

$\mathrm{P}$ HASE unbalance is widespread in distribution networks in the UK, continental Europe, US, China, and other countries. Three-phase current data from a sample of 800 representative low voltage (LV) substations in South Wales, UK, show that more than $50 \%$ of these networks suffer from significant phase unbalance [1]. For instance, it is common that one phase has a peak current of 300A and another phase only has 150A. Main causes for phase unbalance are: 1) an uneven allocation of single-phase loads/customers across the three phases [2], [3], [4]; 2) random load behavior [2], [5]; 3) network structural asymmetries, e.g. the existence of single-phase laterals [6] and unequal phase impedances [7], [8]; and 4) the occurrence of unbalanced faults [9]. Even if the three phases of a distribution network were balanced when the network was initially constructed, phase unbalance appears and accumulates over time with loads being added to and removed from each phase. Phase unbalance causes a number of consequences: 1) additional network investment costs because of the inefficient use of network assets [10], [11], [12]; 2) extra energy losses in the transformer, on the phases and neutral, and in the ground [12], [13], [14]; 3) nuisance tripping [15]; and 4) damages to induction motors [16].

This paper performs a comprehensive review of the scale of phase unbalance, its causes, consequences, and phase rebalancing solutions:

1) This paper analyzes the dominant consequence out of two unbalance-induced consequences (capacity waste and energy losses) for distribution networks of different loading levels.

2) This paper suggests three directions to address the scalability (towards millions of LV networks), data scarcity, and adaptability (unbalance changes over time) challenges arising from phase rebalancing: i) using retrofit-able, maintenance-free, automatic solutions to overcome the scalability challenge; ii) using data analytics to overcome the data-scarcity challenge; and iii) using phase balancers or other online phase rebalancing solutions to overcome the adaptability challenge.

3) This paper analyzes the advantages and limitations of existing phase rebalancing solutions in terms of: i) the suitability for non-directional phase unbalance; ii) energy losses arising from power electronics devices; iii) impact on power supply reliability; iv) cost and scalability to millions of networks; v) the ability to overcome data scarcity; and vi) adaptability towards changing unbalance conditions. This paper also suggests ways to overcome the above limitations.

4) This paper recommends future research topics: i) longterm forecast of phase unbalance; ii) whole-system analysis of the unbalance-induced costs; iii) phase unbalance diagnosis for data-scarce LV networks; iv) techno-commercial solutions to exploit the flexibility from large three-phase customers for phase balancing; v) the optimal placement of phase balancers; vi) the transition from single-phase customers to three-phase customers.

In particular, this paper makes original contributions by providing critical analyses of the existing phase balancing solutions and new insights into future research directions.

The remainder of this paper is organized as follows: Section 
II reviews the scale of phase unbalance; Section III summarizes the consequences of phase unbalance; Section IV reviews popular phase rebalancing solutions and analyzes their pros and cons; Section V recommends future research topics.

\section{Status Quo of Phase Unbalance: Widespread NATURE AND SEVERITY}

Phase unbalance involves both voltage unbalance and current unbalance. IEEE, IEC, National Electrical Manufacturers Association (NEMA), and CIGRE provided different mathematical definitions for voltage unbalance, using either symmetrical components or the minimum, maximum, and average values [17], [18], [19]. IEC defined current unbalance factor as the ratio of the negative sequence component to the positive sequence component [18], [19]. As the consequence of voltage and current unbalance, the power values on the three phases are also unbalanced. Different types of unbalance are coupled with each other and they are likely to occur simultaneously.

\section{A. Phase unbalance in the $U K$}

Phase unbalance is widespread in the UK's distribution networks. The University of Bath have time-series phase current data collected from 800 data-rich LV substations throughout a year at an interval of 15 minutes. These substations cover a good mix of geographical areas (urban, suburban, and rural) and customer composition (domestic, commercial, and industrial) in South Wales, UK. For example, Cardiff city center is selected as an urban area with a large number of commercial customers; Monmouthshire is selected as a representative rural area [1]. These data show that more than $50 \%$ of the $800 \mathrm{LV}$ substations suffer from serious phase unbalance, where the peak current of the "heaviest" phase is greater than that of the "lightest" phase by at least 50\%. TNEI, a UK consultancy company, analyzed a total of $89 \mathrm{LV}$ substations with 233 feeders within the business area of Scottish Power Energy Networks (SPEN) in the UK [15]. They found that 165 out of these $233 \mathrm{LV}$ feeders suffer from significant phase unbalance, where the mean ratio of the phase current to the average current for all three phases is greater than 1.3 [15]. These studies collectively indicate the mass scale of phase unbalance in the UK. The increases of single-phase distributed generation, electric vehicles, and electric heat pumps will likely aggravate phase unbalance in the UK, if they are not properly controlled [20]. L. Ochoa et al. concluded that phase unbalance must be considered for the UK's LV distribution networks [21], which have single-phase laterals supplying single-phase customers [6]. At the medium voltage (11 kV and $33 \mathrm{kV})$ level, the assumption of balanced three phases is not realistic but is an acceptable approximation [21].

\section{B. Phase unbalance in continental Europe}

Phase unbalance is also widespread in continental Europe. European LV distribution networks are mainly three-phase four-wire networks, where the three phases have uneven load connections [4]. For example, in Denmark where domestic customers are mainly supplied with three-phase power, distribution system operators do not have control over which phase each appliance is connected to [22]. Electricians make such decisions, which inevitably lead to phase unbalance, because: 1) each appliance demonstrates random load behavior and 2) the phase connection decisions are made locally without coordination with the grid [22].

\section{Phase unbalance in the US}

The US distribution networks have different layouts from their European counterparts: i) the European distribution networks have three-phase MV/LV transformers with capacities from $300 \mathrm{kVA}$ to $1,000 \mathrm{kVA}$. Extended from these transformers, three-phase LV main feeders supply either threephase customers or single-phase laterals where single-phase customers are connected [23]. ii) On the other hand, the US distribution networks have MV main feeders (the primary system) as the backbone. Extending from the MV main feeders are single-phase laterals that interface the MV feeders with single-phase transformers. These single-phase transformers, normally having capacities less than $100 \mathrm{kVA}$, supply singlephase customers via very short (typically no more than $80 \mathrm{~m}$ ) single-phase LV feeders (the secondary system) [23].

Despite the differences in the distribution network layouts, phase unbalance is also widespread in the US distribution networks, especially the MV distribution networks. In the US, the Department of Energy has identified phase unbalance as the leading cause for the overheating and premature failures of induction motors [24], [25]. The fact that motor-driven systems consume more than $50 \%$ of the electrical energy (the percentage is $70 \%$ for industrial plants) [24], alongside the impacts of phase unbalance on motors, increases the significance of phase unbalance on a whole system level. This also makes phase balancing a key measure to improve motor efficiency [26].

\section{Phase unbalance in other countries}

Phase unbalance is rampant in China. The connections of single-phase domestic customers is identified as a major cause for phase unbalance in China's medium and low voltage distribution networks [27], [28]. The increase of single-phase loads, especially nonlinear loads, further aggravates phase unbalance [29]. J. Wang et al studied the phase unbalance in Jiangsu Province, China [30]. Within a ten-day period in 2015, the study recorded a total of 3,762 phase unbalance daily records from 2,432 distribution transformers (a daily record is defined as a day when phase unbalance lasts for more than 6 hours) [30]. W. Li et al investigated the causes and characteristics of phase unbalance in a town [27]. The study identified correlations between phase unbalance and a variety of factors: the season, time, loading level, and the type of customers [27]. In Australia like in other countries, an uneven allocation of single-phase loads causes phase unbalance. The customer side experiences more serious phase unbalance than the substation side [8].

\section{CAUSES For Phase UnBalance}

The main causes for phase unbalance are:

1) Uneven allocations of loads across three phases. In the 
UK, domestic customers account for more than $50 \%$ of the total number of customers and the majority of the domestic customers are connected to single-phase supply along singlephase laterals [6]. These single-phase domestic customers are allocated to the three phases in an uneven fashion. Even if customers were allocated evenly when the distribution network was constructed, their phase connectivity record may not be properly maintained over decades. When new customers are connected, distribution network operators simply connect them to a random phase for convenience [22], causing inevitable phase unbalance. This gives rise to phase identification techniques [31], which incur costs. In other countries, even if all customers including domestic ones are connected to threephase supply, they have single-phase appliances that are unevenly allocated across three phases, e.g. in Denmark as mentioned above [22]. The phase connectivity of single-phase appliances are not likely to be controlled or even known by network operators.

2) Structural asymmetries over three phases. It is common that single-phase laterals extend from three-phase LV main feeders in the UK's distribution networks [6]. These singlephase laterals represent structural asymmetries over three phases. Further, W. H. Kersting pointed out that the distribution line segments are inherently asymmetrical [7], causing asymmetrical self and mutual impedances and eventually causing widespread current and voltage unbalances in distribution networks [32].

3) Random customer behavior is another cause for phase unbalance. Even if customers are allocated evenly across three phases, they have different load behavior, causing real-time phase unbalance. The unbalance can be aggravated by increasing connections of single-phase large loads with random behavior, such as electric vehicles (EVs) and electric heat pumps (EHPs). The EV charging behavior depends on customers' life styles and the EHP loads depend on the weather, season, building insulation, and indoor setting, etc. [33].

4) Unbalanced faults are another cause for phase unbalance. The unbalanced faults include line-to-line short circuit, line-toground short circuit, line-to-line-to-ground short circuit, and single-phase broken-line fault, etc. Symmetrical components are typically used for fault analysis. The unbalanced faults are not the focus of this paper as this paper emphasizes phase unbalance under normal circumstances.

\section{Consequences of Phase Unbalance}

Four main consequences of phase unbalance are reviewed:

1) Network capacity waste and the associated investment costs

A main consequence arising from phase unbalance is a waste of network capacity and the associated cost [11], [34]. Phase unbalance wastes the capacity of LV feeders. For a feeder with unbalanced three phases, when the capacity of the heaviest phase is used up, the space capacity of the other two phases cannot be transferred to the heaviest phase, thus resulting in network investment being earlier than if the three phases were balanced [10]. Additional investment costs arise from such an unbalance-induced capacity waste. The costs are estimated for both individual networks [10], [35] and the entire business area of a distribution network operator [36]. Phase unbalance is a limiting factor for the connection of distributed generation [20].

\section{2) Unbalance-induced energy losses}

Another main consequence from phase unbalance is additional energy losses, including the additional loss on the phases and that in the neutral and ground (if the neutral is earthed). The additional energy losses occur on both transformers [37], [38] and feeders [39], [40]. J Watson et al presented a method for the optimal dispatch of energy storage to minimize power losses on both the phases and the neutral [41]. W. H. Kersting et al developed a method for computing the currents in the neutral and dirt as well as the associated power losses, which are the consequence of phase unbalance [7]. L. Fang and K. Ma developed a statistical approach to estimate the unbalance-induced energy losses in the neutral and dirt for data-scarce LV networks with data that are only recorded once a year [13]. S. Pajic et al found that for a wide range of conditions, the ratio $\rho$ between the neutral path resistance and the resistance of a transposed three-phase line is approximately given by $\rho \approx R_{0} / R_{1}$, where $R_{0}$ and $R_{1}$ are the zero and positive sequence resistances of the phase wire, respectively [14]. This important conclusion facilitates an efficient estimation of the power loss on the neutral path [14]. Egwaile et al estimated the unbalance-induced energy loss in distribution transformers [39]. The case studies found that rebalancing the loads across the three phases could reduce the transformer energy loss by $6 \%$ [39].

\section{3) Unbalance-induced nuisance tripping}

Nuisance tripping is another consequence from phase unbalance. The ground current caused by phase unbalance may trip the protection devices [34]. Phase current unbalance can cause nuisance tripping to a variable-frequency drive (VFD), even when the average phase power is below the VFD current rating [42].

\section{4) Motor overheating and damages}

Phase unbalance is widely acknowledged to cause motor overheating and even damages. As explained in Section II - C, the US Department of Energy identified phase unbalance as the leading cause for the overheating and premature failures of induction motors [24], [25]. The negative-sequence reactance of an induction motor is only $1 / 7$ to $1 / 5$ of its positive-sequence reactance [43]. The consequence is that, a negative-sequence voltage of $1 \%$ corresponds to a negative-sequence current of $6 \%$, which causes extra heating to the motor and energy losses.

Fig. 1 shows the causes for and consequences of phase unbalance.

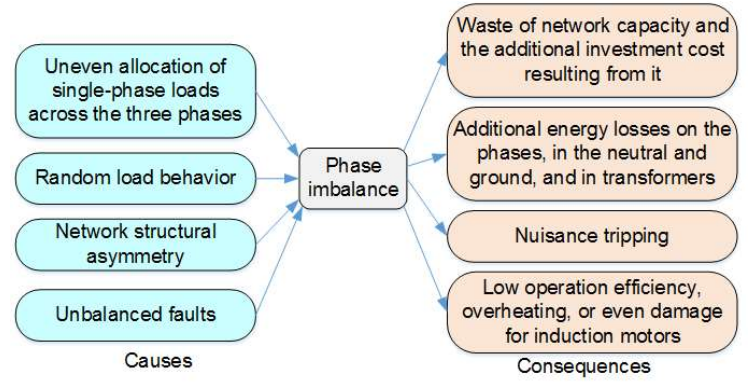


Fig. 1: Causes for and consequences of phase unbalance.

\section{A. Demonstration of phase unbalance consequences}

The degree of phase unbalance for three-phase feeders is defined in [10]. The unbalance-induced cost of capacity wastes (also known as the additional reinforcement cost) on a threephase feeder is given by [10]

$$
A R C=C(1+d)^{\frac{\log U}{\log (1+r)}}\left[(1+d)^{\frac{\log \left(3 D_{\mathrm{IB}}+1\right)}{\log (1+r)}}-1\right]
$$

where $C$ denotes the future cost of feeder replacement; $d$ is the discount rate; $r$ is the annual load growth rate; $U$ is the feeder's three-phase overall loading level, also the nominal loading level; $D_{\mathrm{IB}}$ is the feeder's degree of phase unbalance.

This paper compares the unbalance-induced cost of capacity wastes versus the additional energy losses (the latter is calculated for one year) and identifies the dominant one for urban and suburban distribution networks.

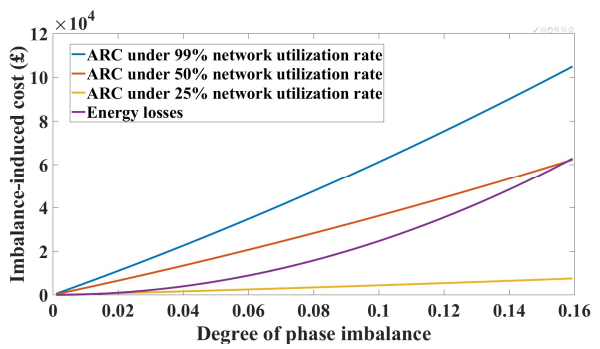

Fig. 2: Comparison of unbalance-induced energy losses and additiona reinforcement costs (ARC) for urban networks

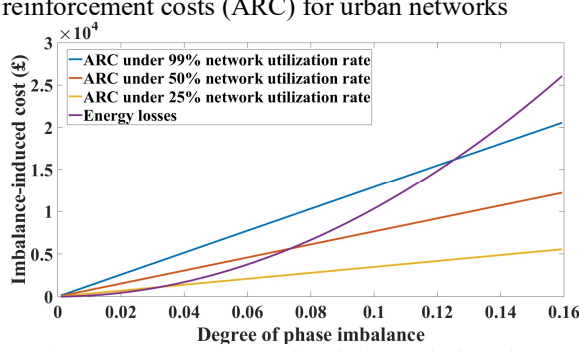

Fig. 3: Comparison of unbalance-induced energy losses and additional reinforcement costs (ARC) for sub-urban networks

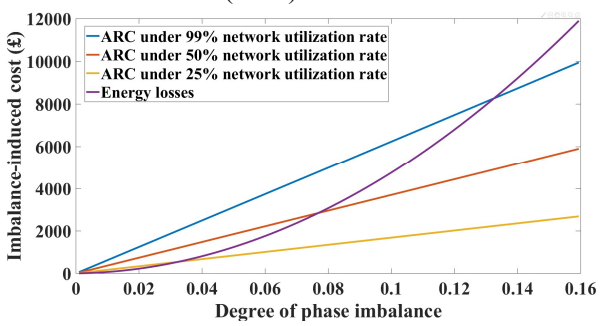

Fig. 4: Comparison of unbalance-induced energy losses and additiona reinforcement costs (ARC) for rural networks

With the increase of degree of phase unbalance, the capacity waste, which is represented by additional reinforcement cost (ARC), dominates the main consequence of phase unbalance for urban networks. However, for sub-urban networks, the dominance changes from capacity waste (represented by the ARC) to energy losses. For sub-urban networks with a loading level of $99 \%$, the threshold degree of phase unbalance is 0.13 , where the cost of capacity waste is equal to that of extra energy losses. The threshold degrees of phase unbalance are 0.058 and 0.023 , respectively, for networks with loading levels of $50 \%$ and $25 \%$. For rural LV networks, the conclusion is similar to that for sub-urban networks. The threshold degrees of phase unbalance are $0.13,0.076$ and 0.024 , respectively, for networks with loading levels of $99 \%, 50 \%$ and $25 \%$.

Reference [36] developed formulas to compute the costs of unbalance-induced network capacity wastes throughout a utility scale. The utility-scale cost of LV feeders' capacity wastes is given by [36]:

$$
A R C_{f}=N_{f} C_{f} \int_{0}^{100 \%} f_{u}(U)\left[\int_{g(U)}^{d_{I B_{-} f \max }} F_{D I B}(x) d x\right] d U
$$

where $g(U)=\min \left\{\frac{1}{3 U}-\frac{1}{3}, \frac{2}{3}\right\}$

In (2), $N_{f}$ denotes the total number of feeders within the region in question; $C_{f}$ is the average investment cost of a feeder; $U$ denotes a feeder's three-phase overall loading level, also the nominal loading level; $f_{u}(U)$ is the probability density function of the nominal loading levels; $g(U)$ is defined in (14); $d_{I B_{-} \text {fmax }}$ is the maximum degree of phase unbalance among all feeders; $F_{D I B}(x)$ is the probability density function of the feeders' degrees of phase unbalance values.

\section{Existing Solutions to Phase Unbalance}

Three challenges arise from phase rebalancing: 1) scalability - millions of LV networks need to be balanced; 2) data scarcity - the majority of the LV networks either do not have any available data or have data that are collected only once a year or at an even lower frequency; 3) adaptability - the phase unbalance condition changes over time with new connections of large single-phase loads such as electric vehicles and heat pumps.

Existing phase rebalancing solutions are classified into three categories and their performance with respect to the above challenges are summarised:

\section{1) Re-phasing of loads and/or laterals}

This type of solution refers to moving loads/laterals from the heavily loaded phase to the light phase(s) either when energized ("online") or during scheduled power cuts ("offline"). Also known as phase swapping, this is a traditional and intuitive way of phase rebalancing. J. Zhu et al formulated the re-phasing problem as an optimal power flow (OPF) problem, where: the control variables are the re-phasing options at each candidate nodes; the objective is to minimize the weighed sum of the rephasing cost and the cost of phase unbalance [11]. Simulated annealing was adopted to solve the nonlinear OPF problem [11]. In [34], J. Zhu et al developed a mixed-integer optimization model for re-phasing, considering both nodal rephasing and lateral re-phasing. D. Singh et al incorporated the voltage-dependency of loads into an online re-phasing model, which is a mixed-integer nonlinear optimization model [44]. W. M. Siti et al developed a combined heuristic and neural network approach, which both reconfigures the feeders and performs customer re-phasing through switching [2]. The same authors also developed a combined fuzzy logic and Newton-Raphson method to re-phase customers on the secondary feeder, with 
fuzzy objectives to minimize a number of phase unbalance indicators [45]. C. Lin et al developed an expert system based on heuristics to re-phase laterals and single-phase transformers, with the objective to reduce the neutral current [46]. T. H. Chen et al developed a genetic-algorithm-based approach to achieve both phase rebalancing and loss reduction for radial distribution networks, where the control variables are the phase connectivity of the transformers connected to a primary feeder [47]. K. Wang et al applied six algorithms to the phase rebalancing problem and identified dynamic programming as a promising algorithm because of its optimality [48]. S. H. Soltani et al developed a dynamic re-phasing strategy, which automatically re-phases loads at any time of a day when the degree of unbalance exceeds a threshold through automatic re-phasing switches [49]. O. Homaee et al developed a phase balancing approach that optimally re-phases single-phase customers using discrete genetic algorithm [50].

Limitations of re-phasing, challenges, and potential solutions are:

i) Only for directional phase unbalance: offline re-phasing is only applicable to phase unbalance with a definite direction, i.e. the existence of a phase that is consistently heavier (or lighter) than the other phases.

ii) Data-scarcity: in LV networks, the time-varying phase unbalance characteristics and customers' phase connectivity are not known. A potential solution is to deploy low-cost monitoring devices at a set of representative LV networks and then use machine learning to extrapolate the knowledge from representative data-rich samples to the vast population of datascarce networks. This type of solution can be applied to identifying customers' phase connectivity [31], the estimation of unbalance-induced energy losses [13], the development of re-phasing guidance [51], etc.

iii) Scalability: digging up roads and re-phasing the cables for each individual LV network are impractical for millions of networks on a utility scale. A potential solution is to identify a small percentage of LV networks with serious phase unbalance out of millions of networks and then only target the identified ones.

iv) Adaptability: the random new connections of large singlephase loads (e.g. electric vehicles) can completely change the direction of phase unbalance, invalidate any previous offline rephasing effort, and cause phase unbalance to reappear. Offline re-phasing is unable to address this problem. Online re-phasing is capable of resolving this, but frequent switching increases energy losses as well as wear and tear.

\section{2) Using phase balancers based on power electronics}

S. M. Fazeli developed an individual-phase decoupled PQ control logic for distributed Static Synchronous Compensator (DSTATCOM) to rebalance the active power and the voltages of the three phases [52], [53]. S. Yan et al developed a new three-phase electric spring and its control logic for balancing the three-phase load of a smart building [3]. F. Shahnia et al developed optimal placement and control methods for a DSTATCOM to perform phase rebalancing [54]. A. Alsulami et al evaluated the dynamic performance of a Static Var Compensator (SVC) in mitigating the negative sequence component of the network voltage/current [55]. H. Chiu et al developed a current-sharing method for a three-phase power factor correction converter to eliminate current unbalance [56]. S. Beharrysingh developed a static balancer, which is essentially an interconnected-star autotransformer, for phase rebalancing in LV networks [12]. S. Liu et al developed a practical online phase switching method to mitigate phase unbalance based on data-driven customer phase identification [57], essentially developing an integrated phase identification and online switching technique.

An advantage of phase balancers is their adaptability towards changing unbalance conditions. In other words, phase balancers rebalances the three phases online in response to changing unbalance conditions. However, they have limitations and they also face challenges:

i) Efficiency: phase balancers are based on power electronics that perform high-frequency switches. These devices incur energy losses that cannot be ignored. They typically have an efficiency less than 95\% [58], [59], whereas distribution transformers have an efficiency $>97 \%$ (the efficiency of liquidimmersed transformers is as high as $99.5 \%$ ) [59]. The more advanced control function and the greater time resolution of phase rebalancing, the more complex the topology of a phase balancer is and the greater losses are incurred. The excess energy losses compared to conventional, mature distribution network assets (e.g. transformers) is a fundamental problem for power electronic devices. The fundamental solution is beyond the power system discipline. It requires a breakthrough to future switching devices (e.g. insulated-gate bipolar transistors) that have a very high efficiency and a high number of lifetime switches - but it can be costly when the technology is at an early stage. An alternative solution to improve efficiency is to reduce the switching frequency, for example, by switching phases only when both the load current and the phase unbalance degree are above predefined thresholds - only the "peak load with a high degree of phase unbalance" case is worth attention.

ii) Reliability: phase balancers connected in series to the grid compromise power supply reliability. The outage of a seriesconnected phase balancer causes an outage of its downstream network. The outage lasts until either the fault is cleared or the downstream network is reconfigured. Potential solutions are: using phase balancers that are connected in parallel to the grid, maintaining redundancy, using components of a high reliability, and using as simple a hardware structure as possible. However, redundancy adds to the cost.

iii) Cost and scalability: the costs of phase balancers include capital and installation costs, costs of monitoring and communication, and maintenance costs. To reduce the total cost, this paper recommends the deployment of retrofit-able, maintenance-free, automatic phase balancers. They can be retrofitted to the existing network, requires minimal or no maintenance, and performs automatic phase rebalancing.

3) Controlling energy storage (including electric vehicles), distributed generation, and micro-grid for phase rebalancing

$\mathrm{S}$ Chen et al developed a game theoretic approach for phase balancing, where a non-cooperative game incentivizes plug-in electric vehicles (EVs) to charge in a way that benefits both the 
TABLE I: SUMMARY OF PHASE REBALANCING SOLUTIONS

\begin{tabular}{|c|c|c|c|}
\hline Solutions & Advantages & Limitations and challenges & $\begin{array}{c}\text { Ways to overcome limitations and } \\
\text { challenges }\end{array}$ \\
\hline $\begin{array}{l}\text { 1. Re-phasing } \\
{[11],[34],[44],[2],} \\
{[45], \quad[46], \quad[47],} \\
{[48],[49],[50]}\end{array}$ & Intuitive & $\begin{array}{l}\text { 1. Offline re-phasing is only applicable to phase } \\
\text { unbalance with a definite direction } \\
\text { 2. Data scarcity challenge: the lack of sensory } \\
\text { data in LV networks poses a challenge to re- } \\
\text { phasing } \\
\text { 3. Scalability challenge: lack of scalability for } \\
\text { millions of LV networks } \\
\text { 4. Adaptability challenge: not adaptive towards } \\
\text { changes of phase unbalance }\end{array}$ & $\begin{array}{l}\text { Data analytics to extrapolate knowledge } \\
\text { from representative data-rich networks to } \\
\text { the cast population of data-scarce networks } \\
\text { Only perform re-phasing for a subset of LV } \\
\text { networks that have a serious phase } \\
\text { unbalance } \\
\text { Offline re-phasing is unable to address this. } \\
\text { Online re-phasing can address this by } \\
\text { frequent switching. }\end{array}$ \\
\hline $\begin{array}{l}\text { 3. Control energy } \\
\text { storage, EVs, DG, } \\
\text { and micro-grids } \\
{[60], \quad[61], \quad[62] \text {, }} \\
{[22], \quad[63], \quad[64],} \\
{[65],[66]}\end{array}$ & $\begin{array}{l}\text { Adaptive; no new } \\
\text { phase } \\
\text { required }\end{array}$ & $\begin{array}{l}\text { Costs of implementation, operation, and } \\
\text { maintenance; scalability issue when applied to } \\
\text { millions of LV networks }\end{array}$ & $\begin{array}{l}\text { Use mature and low-cost technologies for } \\
\text { control and communication. Perform } \\
\text { automatic control }\end{array}$ \\
\hline
\end{tabular}

grid and EV owners [60]. S. Sun et al developed control algorithms for energy storage to balance the three phases under uncertainties [61]. T. Hong et al developed centralized operation schemes for DC micro-grids to: i) fully rebalance the three phases of the substation transformer during the peak load period; and ii) partially rebalance the three phases if the microgrid capacity is insufficient [62]. P. Lico et al developed a control algorithm which coordinates and controls single-phase EV chargers for phase rebalancing [22]. K. H. Chua et al developed a control strategy for energy storage as part of the single-phase photovoltaic system to perform phase rebalancing [63]. C. C. Bajo et al developed an individual-phase control strategy for three-phase PV systems to perform phase rebalancing [64]. F. Shahnia et al developed a control method for single-phase PV converters to provide reactive power support, thus reducing voltage unbalance [65]. E. VegaFluentes et al developed a coordination strategy for singlephase EV charging with three-phase power supply: when charging starts, chargers connect EVs to the lightly loaded phase of their feeder [66]. This would reduce unbalanceinduced consequences and increases the network capacity to host EVs.

An advantage of this type of solutions is that no additional phase balancer is required. However, their costs and scalability are still a concern: the installation, communication, operation, and maintenance costs for millions of LV networks. In particular, price- or market-driven solutions (such as electric vehicle smart charging) incur transaction costs that may discourage the direct participations of domestic customers. These costs should be factored into the cost-benefit analysis of the phase balancing solution.

Table I summarizes the phase rebalancing solutions, which not only reduce energy losses but also release extra network capacities that are otherwise wasted by phase unbalance. This paper suggests the following directions that will address the scalability, data-scarcity, and adaptability challenges arising from phase rebalancing: 1) use retrofit-able, maintenance-free, automatic solutions to overcome the scalability challenge; 2 ) use data analytics to overcome the data-scarcity challenge by extrapolating knowledge to data-scarce networks; and 3) use phase balancers or other online phase rebalancing solutions to overcome the adaptability challenge.

\section{FUTURE RESEARCH TOPICS}

A number of promising future research topics are suggested:

1) Long-term forecast of phase unbalance. It is yet an unresolved question to perform a long-term forecast of the 
phase unbalance degree for years into the future. Traditional forecast methods are based on historical data [30]. However, historical data do not reflect future increases of disruptive loads such as electric vehicles and electric heat pumps, especially if they are single-phase connected. Therefore, projections solely based on historical data are questionable. The key to the longterm phase unbalance forecast is to model in a stochastic way both the overall growth of those disruptive loads, their behavior and phase connectivity. The last one refers to the stochastic allocation of disruptive loads across the three phases, given existing phase connectivity information, e.g. the customer mix (domestic, industrial, and commercial customers) of each phase. It is expected that with these disruptive loads, the fluctuations of phase unbalance will be aggravated. However, how the degree of phase unbalance will change is uncertain, depending on how balanced the disruptive loads will increase across three phases and depending on whether distributed generation matches electric vehicles over time. In this process, it is possible that the variations of phase unbalance as well as the peak phase unbalance will increase, although the average degree of phase unbalance over a year may not increase.

Scenario-based long-term phase unbalance forecasts are needed on a range of representative distribution networks. Probability distributions and Monte-Carlo simulations are required to model and simulate the uncertainties associated with load/generation growth, behavior, and phase connectivity. The forecast will determine the projected costs of phase unbalance for distribution network operators. These costs will be translated into the potential benefits from phase rebalancing and will determine whether it is economically feasible for distribution network operators to invest in phase balancing solutions.

2) Whole-system analysis of the unbalance-induced costs. Whole-system analysis of the costs of phase unbalance, considering the propagation of unbalance from the very root (i.e. customers) to LV, medium voltage (MV), and high voltage (HV) distribution networks. Although the degree of phase unbalance is expected to decrease with the increase of the voltage level because of the load aggregation effect, the unbalance-induced costs cannot be simply ignored at MV and HV distribution levels. This is because the unbalance-induced network investment costs are proportional to the asset capital costs, which are greater at MV and HV levels than at the LV level. It is expected that the unbalance-induced capacity wastes will be a main concern, whereas the unbalance-induced energy losses will quickly diminish at higher voltage levels. For example, an $11 \mathrm{kV}$ distribution network has near-overload cables and transformers and suffers from significant phase unbalance, in which case the unbalance-induced network investment cost should be considered. Previous cost-benefit analysis of phase balancing solutions focused on LV networks only [67], resulting in an underestimation of the phase balancing benefits and thus a conservative cost-benefit analysis. A cross-voltage-level analysis would reveal a more accurate cost of phase unbalance (also the potential benefit from phase rebalancing) than the analysis solely focusing on the LV level, thus enabling a more accurate cost-benefit analysis of phase rebalancing solutions. This will guide distribution network operators to make informed decisions on whether it is better to leave phase unbalance as it is or to invest in phase rebalancing solutions (as well as the type of solutions).

The key to the whole-system analysis of phase unbalance is to model the upward propagation of phase unbalance, i.e. how quickly phase unbalance diminishes upwards from LV to MV or HV levels for distribution networks that may or may not have sufficient sensory data.

3) Phase unbalance diagnosis for data-scarce LV networks. A promising topic is to diagnose phase unbalance for datascarce LV networks, including the diagnosis of phase unbalance direction and its coincidence with peak loads.

The existence of a phase unbalance direction refers to the existence of a particular phase that is consistently heavier (or lighter) than the other two phases. An example of phase unbalance with a definite direction is: phase a is heavier than the other two phases for $70 \%$ of the time and that phase a has an average current greater than that of the other phases. It is important to identify whether a phase unbalance direction exists or not, because the existence of the direction indicates that the loads are unevenly allocated towards a particular phase [68], meaning that offline load re-phasing is a feasible solution, e.g. move loads away from the consistent heavy phase to the other two phases during scheduled power cuts [68]. However, not all cases have such a direction, e.g. a network where each phase is the heaviest phase for $1 / 3$ of the time and the three phases have roughly equal average currents. In such cases, random load behavior is the dominant cause for phase unbalance. Solutions other than load re-phasing should be sought for, e.g. deploying phase balancers.

It is also important to diagnose to what extent phase unbalance coincides with the peak demand. If the peak demand coincides with the maximum degree of phase unbalance and if the peak demand is already close to the network capacity, the unbalance-induced capacity waste would be aggravated. Such a coincidence indicates that phase rebalancing is particularly urgent. On the other hand, if the peak demand does not coincide with the peak degree of phase unbalance, the unbalanceinduced energy losses would be a main consequence.

A promising research topic is to develop data-efficient (require a minimal amount of data) methods to diagnose phase unbalance for data-scarce LV networks. Ideally, the methods shall utilize the data that can be easily obtained during lowfrequency (e.g. once a year) inspections of the networks/substations by field engineers. The key is to extract knowledge from representative data-rich networks and extrapolate the knowledge to the data-scarce networks, using a combination of signal processing and data analytics.

An alternative approach to data analytics is to design and deploy low-cost sensors to collect phase currents and voltages data, with built-in function to detect phase unbalance. In order for the sensors to be scalable towards millions of LV networks, these sensors should ideally be free from transformers, be able to process information locally with minimal communications, be maintenance-free, and ideally be integrated into existing 
substation-level energy meters. These sensors will support the diagnosis of phase unbalance.

4) Techno-commercial solutions to exploit the flexibility from large three-phase customers for phase rebalancing. There is a potential to exploit large three-phase customers (parking lots, building) for phase balancing through appropriate incentive schemes. Making three-phase customers draw balanced three-phase power does not aggravate the existing phase unbalance in the network, but it does not contribute to phase rebalancing either. This paper argues that, by deliberately making the three phases of large customers unbalanced, it can counter the phase unbalance at the substation. For example, a $11 \mathrm{kV} / 415 \mathrm{~V}$ substation has instantaneous load currents of $150 \mathrm{~A}$, $100 \mathrm{~A}, 70 \mathrm{~A}$ on phases a, b, and c, respectively. At this instance, a three-phase load which interfaces the network with power electronics can be controlled to draw unbalanced three-phase currents that counter the phase unbalance at the substation, e.g. 0,0 , and $30 \mathrm{~A}$ on phases $\mathrm{a}, \mathrm{b}$, and $\mathrm{c}$, respectively, at the point of common coupling. This is "better" for the substation in terms of phase balancing, compared to the case where the three-phase load draws a current of $10 \mathrm{~A}$ on each phase. This requires realtime automatic communication and control between the substation and the customer. The key technical part of the solution includes: 1) developing a control strategy to allocate the substation-side phase rebalancing task among all contracted customers/aggregators in the form of a control objective for each customer/aggregator; and 2) developing a control logic for contracted customers to perform phase current control to meet the control objective mentioned in 1).

The commercial part of the phase rebalancing solution is to develop a commercial arrangement to incentivize large threephase customers to contribute to phase rebalancing. This requires an estimation of the unbalance-induced cost, which is translated into the potential gross benefit from phase rebalancing. The net benefit is the gross benefit less the implementation cost. The distribution network operator needs to share the net benefit among all contributing customers, e.g. in the form of electricity bill discounts. Furthermore, the phase rebalancing service can be integrated with demand-side response services, referred to as "revenue stack up", to relieve the stress and improve operation efficiency for the network.

5) The optimal placement of phase balancers. A phase balancer can be deployed at different candidate locations of a network, e.g. the substation, a feeder node, and the customer side. Deployments at different locations have their respective pros and cons. If a phase balancer is deployed at a three-phase feeder node close to customers or at the three-phase customer's point of common coupling (PCC), it eliminates the unbalance close to its very source, i.e. customers, but new unbalance can emerge upstream of the phase balancer. On the other hand, if a phase balancer is deployed at the MV/LV substation or even at a higher-level substation, it does not reduce any downstream unbalance on the feeders but it effectively prevents the unbalance from propagating upwards.

Further, the more upstream (closer to the LV substation or at the MV level) a phase balancer is deployed, the more it serves the purpose of saving unbalance-induced network investment costs and the less it serves the purpose of reducing unbalanceinduced energy losses. This is because unbalance-induced energy losses mainly occur on LV feeders. This has implications on phase balancer placement. If a LV feeder has a significant capacity margin and the purpose of phase balancing is to reduce energy losses, then phase balancers should be deployed close to customers, i.e. the source of unbalance.

In all cases, the optimal placement of a phase balancer requires a cost-benefit analysis, which can be formulated as a mixed-integer optimization model, where the decision variable is the optimal location of the phase balancer.

6) The transition from single-phase customers to threephase customers. Unlike in continental Europe, the majority of the UK's existing customers have single-phase supply. A recent study advocated that new customers should be supplied with three-phase power and investigated the feasibility to retrofit three-phase supply to existing single-phase domestic customers [69]. The purpose for the change is to increase the supply capacity to accommodate new electric vehicles, electric heat pumps, and photovoltaic generation with a capacity of greater than $4 \mathrm{~kW}$. If these large loads and distributed generation will be balanced three-phase loads, the overall degree of phase unbalance (relative to the loading level) is expected to decrease as compared to the scenario where these loads and distributed generation are single-phase connected. However, the impact of such a transition on phase unbalance needs to be quantified and this should be factored into the cost-benefit analysis of the transition from single-phase customers to three-phase customers.

\section{REFERENCES}

[1] "Demonstration of LV Network Templates through statistical analysis," https://www.westernpower.co.uk/downloads/5050.

[2] M. W. Siti, D. V. Nicolae, A. A. Jimoh, and A. Ukil, "Reconfiguration and Load Balancing in the LV and MV Distribution Networks for Optimal Performance," Power Delivery, IEEE Transactions on, vol. 22, no. 4, pp. 2534-2540, 2007.

[3] S. Yan, S. C. Tan, C. K. Lee, B. Chaudhuri, and S. Y. R. Hui, "Electric Springs for Reducing Power Imbalance in Three-Phase Power Systems," IEEE Transactions on Power Electronics, vol. 30, no. 7, pp. 3601-3609, 2015.

[4] J. Nájera, H. Mendonça, R. M. de Castro, and J. R. Arribas, "Strategies Comparison for Voltage Unbalance Mitigation in LV Distribution Networks Using EV Chargers," Electronics, vol. 8, no. 3, pp. 289, 2019.

[5] G. Mokryani, A. Majumdar, and B. C. Pal, "Probabilistic method for the operation of three-phase unbalanced active distribution networks," IET Renewable Power Generation, vol. 10, no. 7, pp. 944-954, 2016.

[6] V. Rigoni, L. F. Ochoa, G. Chicco, A. Navarro-Espinosa, and T. Gozel, "Representative Residential LV Feeders: A Case Study for the North West of England," IEEE Transactions on Power Systems, vol. 31, no. 1, pp. 348-360, 2016

[7] W. H. Kersting, "The computation of neutral and dirt currents and power losses." pp. 213-218 vol.1.

[8] F. Shahnia, A. Ghosh, G. Ledwich, and F. Zare, "Predicting Voltage Unbalance Impacts of Plug-in Electric Vehicles Penetration in Residential Low-voltage Distribution Networks," Electric Power Components and Systems, vol. 41, no. 16, pp. 1594-1616, 2013/12/10, 2013.

[9] R. G. Harley, E. B. Makram, and E. G. Duran, "The effects of unbalanced networks and unbalanced faults on induction motor transient stability," IEEE Transactions on Energy Conversion, vol. 3, no. 2, pp. 398-403, 1988

[10] K. Ma, R. Li, and F. R. Li, "Quantification of Additional Asset Reinforcement Cost From 3-Phase Imbalance," Ieee Transactions on Power Systems, vol. 31, no. 4, pp. 2885-2891, Jul, 2016. 
[11] J. Zhu, G. Bilbro, and M.-Y. Chow, "Phase balancing using simulated annealing," Power Systems, IEEE Transactions on, vol. 14, no. 4, pp 1508-1513, 1999.

[12] S. Beharrysingh, "Phase unbalance on low-voltage electricity networks and its mitigation using static balancers," Loughborough University, 2014.

[13] L. Fang, K. Ma, R. Li, Z. Wang, and H. Shi, “A Statistical Approach to Estimate Imbalance-Induced Energy Losses for Data-Scarce Low Voltage Networks," IEEE Transactions on Power Systems, pp. 1-1, 2019.

[14] S. Pajic, and A. E. Emanuel, "Effect of Neutral Path Power Losses on the Apparent Power Definitions: A Preliminary Study," IEEE Transactions on Power Delivery, vol. 24, no. 2, pp. 517-523, 2009.

[15] "HV and LV Phase Imbalance Assessment "; https://www.spenergynetworks.co.uk/userfiles/file/HVandLVPhaseImbal anceAssessment16.pdf.

[16] A. Jalilian, and R. Roshanfekr, "Analysis of Three-phase Induction Motor Performance under Different Voltage Unbalance Conditions Using Simulation and Experimental Results," Electric Power Components and Systems, vol. 37, no. 3, pp. 300-319, 2009/02/23, 2009.

[17] "IEEE Standard Definitions for the Measurement of Electric Power Quantities Under Sinusoidal, Nonsinusoidal, Balanced, or Unbalanced Conditions - Redline," IEEE Std 1459-2010 (Revision of IEEE Std 14592000) - Redline, pp. 1-52, 2010.

[18] "Definitions of Voltage Unbalance," IEEE Power Engineering Review, vol. 21 , no. 5, pp. 49-51, 2001

[19] A. K. Singh, G. K. Singh, and R. Mitra, "Some Observations on Definitions of Voltage Unbalance." pp. 473-479.

[20] L. Thomas, "Connection Imbalance in Low Voltage Distribution Networks," Cardiff University, 2015.

[21] L. Ochoa, and J. Quiros-Tortos. "Advanced Modelling of Smart Distribution Networks Using OpenDSS," https://www.researchgate.net/profile/Luisnando_Ochoa/publication/2834 53198 Tutorial_ISGT_Latin_America_2015_Advanced_Modelling_of Smart Distribution Networks Using OpenDSS/links/5638aa2208ae51c $\operatorname{cb} 3 \operatorname{cc} \overline{8} 31 \mathrm{f} /$ Tutorial-ISGT-Latin-America-2015-Advanced-Modelling-ofSmart-Distribution-Networks-UsingOpenDSS.pdf?origin=publication list.

[22] P. Lico, M. Marinelli, K. Knezović, and S. Grillo, "Phase balancing by means of electric vehicles single-phase connection shifting in a low voltage Danish grid." pp. 1-5.

[23] E. Csanyi. "North American versus European distribution systems," https://electrical-engineering-portal.com/north-american-versuseuropean-distribution-systems.

[24] "Optimizing your motor-driven system," https://www.energy.gov/sites/prod/files/2014/04/f15/mc-0381.pdf.

[25] "Eliminate Voltage Unbalance," https://www.nrel.gov/docs/fy00osti/27832.pdf.

[26] "Methods to optimize electric motor efficiency," https://www.reliableplant.com/Read/25199/methods-optimize-electricmotor.

[27] W. Li，“桥头低压配电网三相不平衡问题及对策的研究 (English translation: Research in the three-phase imbalance problem in Qiaotou low voltage distribution networks and the solutions) ", South China University of Technology, 2017.

[28] E. Li，“光伏接入用于低压电网三相不平衡治理研究与设计 (English translation: Research and design of phase balancing solution using photovoltaic generation connected to low voltage distribution networks," Shenyang Agricultural University, 2018.

[29] X. Wang, K. Shen, Q. Gao, and X. Fu, “配电网三相不平衡抑制方法 综述 (Review of phase balancing methods for distribution networks), Journal of Shenyang Institute of Engineering(Natural Science), vol. 1, 2016.

[30] W. Jiye, Z. Nan, and H. Hanyong, "Three-phase imbalance prediction: A hazard-based method." pp. 226-231.

[31] M. Xu, R. Li, and F. Li, "Phase Identification With Incomplete Data," IEEE Transactions on Smart Grid, vol. 9, no. 4, pp. 2777-2785, 2018.

[32] W. H. Kersting, Distribution system modeling and analysis, 4th ed.: CRC Press, 2017.

[33] A. Navarro-Espinosa, and P. Mancarella, "Probabilistic modeling and assessment of the impact of electric heat pumps on low voltage distribution networks," Applied Energy, vol. 127, pp. 249-266, 2014/08/15/, 2014.
[34] Z. Jinxiang, C. Mo-Yuen, and Z. Fan, "Phase balancing using mixedinteger programming [distribution feeders]," IEEE Transactions on Power Systems, vol. 13, no. 4, pp. 1487-1492, 1998.

[35] K. Ma, F. Li, and R. Aggarwal, "Quantification of Additional Reinforcement Cost Driven by Voltage Constraint Under Three-Phase Imbalance," IEEE Transactions on Power Systems, vol. 31, no. 6, pp. 5126-5134, Nov, 2016

[36] K. Ma, R. Li, and F. R. Li, "Utility-Scale Estimation of Additional Reinforcement Cost From Three-Phase Imbalance Considering Thermal Constraints," Ieee Transactions on Power Systems, vol. 32, no. 5, pp. 3912-3923, Sep, 2017.

[37] R. Salustiano, E. Tavares, and M. Martinez, The unbalanced load cost on transformer losses at a distribution system, 2013.

[38] A. Njafi, İ. İ, and N. Genc, "Evaluating and derating of three-phase distribution transformer under unbalanced voltage and unbalance load using finite element method." pp. 160-165.

[39] L. F. Ochoa, R. M. Ciric, A. Padilha-Feltrin, and G. P. Harrison, "Evaluation of distribution system losses due to load unbalance," in Power Systems Computation Conference, Liege, 2005.

[40] T.-H. Chen, "Evaluation of line loss under load unbalance using the complex unbalance factor," IEE Proceedings - Generation, Transmission and Distribution, 142, https://digitallibrary.theiet.org/content/journals/10.1049/ip-gtd_19951708, 1995].

[41] J. D. Watson, N. R. Watson, and I. Lestas, "Optimized Dispatch of Energy Storage Systems in Unbalanced Distribution Networks," IEEE Transactions on Sustainable Energy, vol. 9, no. 2, pp. 639-650, 2018.

[42] J. A. DeDad, "Beware Of Line-Current Unbalance On VFDs," Electrical Construction \& Maintenance Magazine, 1999.

[43] "Motor https://www.gegridsolutions.com/multilin/resource/motor/UniFlip_Publi cation $/ \mathrm{html} / 8 . \mathrm{html}$.

[44] D. Singh, R. K. Misra, and S. Mishra, "Distribution system feeder rephasing considering voltage-dependency of loads," International Journal of Electrical Power \& Energy Systems, vol. 76, pp. 107-119, 2016/03/01/, 2016.

[45] W. M. Siti, A. Jimoh, and D. Nicolae, "Distribution network phase load balancing as a combinatorial optimization problem using fuzzy logic and Newton-Raphson," Electric Power Systems Research, vol. 81, no. 5, pp. 1079-1087, 2011/05/01/, 2011.

[46] C. H. Lin, C. S. Chen, H. J. Chuang, M. Y. Huang, and C. W. Huang, "An Expert System for Three-Phase Balancing of Distribution Feeders," IEEE Transactions on Power Systems, vol. 23, no. 3, pp. 1488-1496, 2008.

[47] C. Tsai-Hsiang, and C. Jeng-Tyan, "Optimal phase arrangement of distribution transformers connected to a primary feeder for system unbalance improvement and loss reduction using a genetic algorithm." pp. 145-151.

[48] K. Wang, S. Skiena, and T. G. Robertazzi, "Phase balancing algorithms," Electric Power Systems Research, vol. 96, no. 0, pp. 218-224, 2013.

[49] S. H. Soltani, M. Rashidinejad, and A. Abdollahi, "Dynamic phase balancing in the smart distribution networks," International Journal of Electrical Power \& Energy Systems, vol. 93, no. Supplement C, pp. 374383, 2017/12/01/, 2017.

[50] O. Homaee, A. Najafi, M. Dehghanian, M. Attar, and H. Falaghi, "A practical approach for distribution network load balancing by optimal rephasing of single phase customers using discrete genetic algorithm," International Transactions on Electrical Energy Systems, vol. 29, no. 5, pp. e2834, 2019.

[51] L. Fang, K. Ma, and X. Zhang, "A Statistical Approach to Guide Phase Swapping for Data-Scarce Low Voltage Networks," IEEE Transactions on Power Systems, pp. 1-1, 2019.

[52] S. M. Fazeli, H. W. Ping, N. B. A. Rahim, and B. T. Ooi, "Individualphase decoupled P-Q control of three-phase voltage source converter," IET Generation, Transmission \& Distribution, vol. 7, no. 11, pp. 12191228, 2013.

[53] S. M. Fazeli, H. W. Ping, N. B. A. Rahim, and B. T. Ooi, "Individualphase control of 3-phase 4-wire voltage-source converter," IET Power Electronics, $\quad 7, \quad \mathrm{https} / /$ digitallibrary.theiet.org/content/journals/10.1049/iet-pel.2013.0973, 2014].

[54] F. Shahnia, A. Ghosh, G. Ledwich, and F. Zare, "Voltage Unbalance reduction in low voltage distribution networks with rooftop PVs." pp. 15 .

[55] A. Alsulami, M. Bongiorno, K. Srivastava, and M. Reza, "Balancing asymmetrical load using a static var compensator." pp. 1-6. 
[56] H. Chiu, T. Wang, L. Lin, and Y. Lo, "Current Imbalance Elimination for a Three-Phase Three-Switch PFC Converter," IEEE Transactions on Power Electronics, vol. 23, no. 2, pp. 1020-1022, 2008.

[57] S. Liu, X. Cui, Z. Z. Lin, Z. Lian, Z. Lin, F. Wen, Y. Ding, Q. Wang, L. Yang, R. Jin, and H. Qiu, "Practical Method for Mitigating Three-Phase Unbalance based on Data-Driven User Phase Identification," IEEE Transactions on Power Systems, pp. 1-1, 2020.

[58] C. Xiao, G. Chen, and W. G. H. Odendaal, "Overview of Power Loss Measurement Techniques in Power Electronics Systems," IEEE Transactions on Industry Applications, vol. 43, no. 3, pp. 657-664, 2007.

[59] X. She, A. Q. Huang, and R. Burgos, "Review of Solid-State Transformer Technologies and Their Application in Power Distribution Systems," IEEE Journal of Emerging and Selected Topics in Power Electronics, vol. 1, no. 3, pp. 186-198, 2013.

[60] S. Chen, Z. Guo, Z. Yang, Y. Xu, and R. S. Cheng, "A Game Theoretic Approach to Phase Balancing by Plug-in Electric Vehicles in the Smart Grid," IEEE Transactions on Power Systems, pp. 1-1, 2019.

[61] S. Sun, B. Liang, M. Dong, and J. A. Taylor, "Phase Balancing Using Energy Storage in Power Grids Under Uncertainty," IEEE Transactions on Power Systems, vol. 31, no. 5, pp. 3891-3903, 2016.

[62] T. Hong, F. d. Le, and x00F, "Centralized Unbalanced Dispatch of Smart Distribution DC Microgrid Systems," IEEE Transactions on Smart Grid, vol. 9, no. 4, pp. 2852-2861, 2018

[63] K. H. Chua, J. Wong, Y. S. Lim, P. Taylor, E. Morris, and S. Morris, "Mitigation of Voltage Unbalance in Low Voltage Distribution Network with High Level of Photovoltaic System," Energy Procedia, vol. 12, pp. 495-501, 2011/01/01/, 2011

[64] C. G. Bajo, S. Hashemi, S. B. Kjsær, G. Yang, and J. Østergaard, "Voltage unbalance mitigation in LV networks using three-phase PV systems." pp. 2875-2879

[65] F. Shahnia, R. Majumder, A. Ghosh, G. Ledwich, and F. Zare, "Voltage imbalance analysis in residential low voltage distribution networks with rooftop PVs," Electric Power Systems Research, vol. 81, no. 9, pp. 1805 $1814,2011 / 09 / 01 /, 2011$.

[66] E. Vega-Fuentes, and M. Denai, "Enhanced Electric Vehicle Integration in the UK Low-Voltage Networks With Distributed Phase Shifting Control," IEEE Access, vol. 7, pp. 46796-46807, 2019.

[67] W. Kong, K. Ma, L. Fang, R. Wei, and F. Li, "Cost-Benefit Analysis of Phase Balancing Solution for Data-scarce LV Networks by Cluster-Wise Gaussian Process Regression," IEEE Transactions on Power Systems, pp. $1-1,2020$.

[68] W. Kong, K. Ma, and Q. Wu, "Three-Phase Power Imbalance Decomposition Into Systematic Imbalance and Random Imbalance," IEEE Transactions on Power Systems, vol. 33, no. 3, pp. 3001-3012, 2018

[69] The feasibility, costs and benefits of three-phase power supplies in new homes, Renewable Energy Association, Western Power Distribution, 2018. 